\title{
The frictional force on sliding drops
}

\author{
Joel Koplikw \\ Benjamin Levich Institute and Department of Physics \\ City College of the City University of New York, New York, NY 10031
}

(Dated: August 1, 2021)

\begin{abstract}
The dynamic frictional force between solid surfaces in relative motion differs from the static force needed to initiate motion, but this distinction is not usually thought to occur for liquid drops moving on a solid. Recent experiments [Gao, et al., Nature Phys. 114, 191 (2018)] have challenged this view, and claim to observe an analog of solid-on-solid friction for sliding drops. We use molecular dynamic simulations to investigate the forces that moving liquids exert on solids in several situations. In contrast to the indirect techniques required in laboratory experiments, the forces involved in friction are directly accessible in these calculations. We find that, aside from possible inertial effects due to the abrupt initiation of motion and aging effects for unconfined drops, the frictional forces are constant in time.
\end{abstract}

\section{INTRODUCTION}

Liquids are often distinguished from solids by their response to shear: "A liquid cannot support a shear stress and flows irreversibly and continuously when a stress is applied" (see, e.g., [1]). A partially-wetting drop resting on an ideal, smooth and planar solid surface, held in place by surface tension forces at the contact line, would then begin to slide at once under gravity once the surface tilts. Realistically however [2, the drop would be held in place by surface heterogeneities and only begin to move when a critical tilt angle is reached. At that point, usually characterized in terms of the advancing and receding contact angles, sliding begins. If instead of the liquid a second solid were placed on the surface, once again a critical tilt angle would be needed to initiate motion but here the usual characterization is in terms of solid and dynamic friction. This is a distinction between the force needed to initiate the motion, the point where the ratio of lateral to normal force equals the static friction coefficient, and the lesser force required to sustain the motion, where the ratio is the (smaller) dynamic friction coefficient. Explanations of this phenomenon [3] involve mismatches in the respective surface irregularities, changes in the degree of contact between the two solid surfaces, distortions of the solid lattice and so on. These effects would appear to be absent in the case of a liquid drop, which would adjust itself to achieve complete contact with the solid, and one would not expect a distinction between the forces (tilt angle) needed for the initiation and maintenance of sliding motion.

A recent experiment by Gao et al. 4, concludes that in fact a sliding liquid drop does exhibit distinct static and dynamic friction regimes. In order to measure the force that a sliding drop exerts on a supporting solid experimentally an ingenious indirect technique was developed in which a capillary pin is embedded in a liquid drop placed on a sliding stage and the pin's deflection measured optically as the drop moves past. Calibration of

\footnotetext{
* jkoplik@ccny.cuny.edu
}

pin deflection versus applied force converts the measured defection into the time-dependent force on the pin. The results indicate three regimes: a "static" regime where the drop distorts but moves with the sliding stage, a "threshold" regime where the drop begins to slip and the force on the pin rises to a peak value, followed by a constant-force regime where the drop in held in place by the pin while slipping over the sliding stage. This behavior was observed to be robust in terms of drop and solid materials and pulling speed and appears to be general, although one may wonder about complications due to the motion of the liquid around the pin and the consequent distortion of the drop surface.

These experimental results are quite surprising, and it would be desirable to have them confirmed independently by another technique. While it is difficult to directly access the force exerted on an sliding liquid drop in the laboratory, it is straightforward to do so in a molecular dynamics (MD) simulation. In this calculation, the force between each pair of atoms is computed and it is a simple matter of bookkeeping to identify and isolate the various forces exerted on the drop. In this paper we present the results of MD simulations of several configurations in which liquids move on solids, to test for the presence of any analog to static solid-on-solid friction.

What we actually measure is the force the liquid exerts on a bounding solid. Ostensibly, as the title of the paper suggests, we are interested in the frictional force exerted on the liquid, but the force on the solid is both better defined and more relevant. Instantaneously, the force on the liquid is just equal and opposite to the force on the solid, by Newton's third law, but as discussed below force fluctuations in MD are so severe that it is necessary to average over a finite time interval to obtain a robust value of a force. It is straightforward to follow a region of solid over time, since the structure is fairly rigid, and compute the force on that region, but liquid atoms are subject to diffusion and advection and any liquid region will change its shape and location over time, while any fixed region in a liquid will change its contents. The time-averaged force on the solid is thus well-defined whereas that on a liquid may not be. Furthermore, in any experiment, such 
as the one that motivated this paper, it is the force on a measuring probe that is detected.

We first consider the simpler case of Couette flow initiated in a periodic channel by abruptly translating a bounding wall. This case has two advantages: an analytic solution to the governing equations is available, and there is no moving contact line issue to complicate the problem. This simulation also allows us to identify some general features of force measurement and the initiation of motion, which reappear in drop motion. Next, we study isolated drops sliding on a solid surface due to an applied body force. Here we consider a partially-wetting drop which is equilibrated while resting on an atomistic solid surface and then subjected to a lateral body force, in imitation of a drop placed on a tilted plane in the presence of gravity. We consider both a uniform atomically smooth surface as well as a drop held in place by surface heterogeneity: an abrupt variation in wettability or a step change in surface height. The force measurement results here are quite consistent with the usual description of this flow in terms of dynamic contact angles. Third, as a cleaner analog of the experiment of Gao et al., we study a cylindrical drop placed in the corner where two flat surfaces intersect at $90^{\circ}$, and then translate on of the surfaces so as to drive the drop into the corner. In this way we avoid the complicating effects of distorting the liquid vapor interface as the drop moves around the pin, present in the experiment [4. Lastly we study a direct caricature of the experiment, using a spherical cap droplet on a sliding stage held in place by a fixed pin.

In all of these simulations we find no evidence for enhanced static solid-on-liquid forces beyond the effects of ordinary fluid viscosity and inertia. In some but not all cases we observe a significant peak in the force at early times, but we argue that this is the result of a step change in the force applied to the liquid, which is perfectly well accounted for by the viscous effects built into the Navier-Stokes equations, along with some transient non-Newtonian effects.

\section{COUETTE FLOW}

The simulations are based on classical MD methods [5, 6] and involve a drop composed of a generic Newtonian liquid made of tetramer molecules with LennardJones interactions, adjacent to solid surfaces consisting of atoms tethered to lattice sites. This computational framework is used throughout the paper. The interactions are

$$
\begin{aligned}
V_{\mathrm{LJ}}(r) & =4 \epsilon\left[\left(\frac{r}{\sigma}\right)^{-12}-c\left(\frac{r}{\sigma}\right)^{-6}\right] \\
V_{F E N E}(r) & =-\frac{1}{2} k_{F} r_{0}^{2} \ln \left(1-\frac{r^{2}}{r_{0}^{2}}\right)
\end{aligned}
$$

with parameters $k_{F}=30 \epsilon / \sigma^{2}$ and $r_{0}=1.5 \sigma$ (after Ref. [7. In the following, if not stated explicitly it is
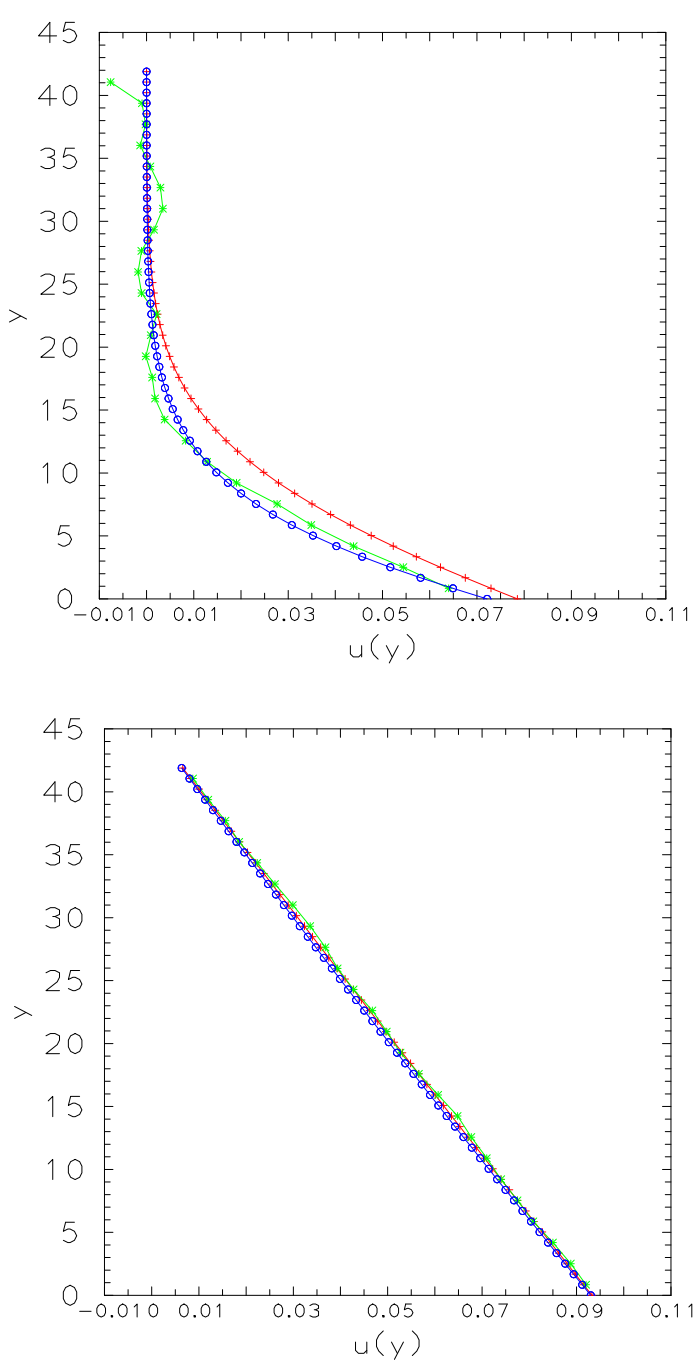

FIG. 1. Couette flow velocity profile at $10 \tau$ (top) and $200 \tau$ (bottom). The MD results (20 realization average) are in green $\left(^{*}\right)$, Newtonian Navier-Stokes in red $(+)$ and shearthinning model in blue (o).

understood that numerical results are given in units of $\sigma$ (length), $\epsilon$ (energy), and $\tau=m(\sigma / \epsilon)^{1 / 2}$ (time), where $m$ is the common atomic mass. The parameter $c$ is used to adjust the strength of the liquid-solid interaction and hence the wettability, as described below. $V_{L J}$, which is cut off at $r=2.5 \sigma$, acts between each pair of atoms and $V_{F E N E}$ acts between successive atoms in a four-monomer linear chain. The solid atoms are bound to lattice sites using a harmonic potential with stiffness $100 \epsilon / \sigma^{2}$ and a local Nosé-Hoover thermostat fixes the temperature at $T=0.8 \epsilon / k_{B}$. This particular liquid-vapor-solid system has been used extensively in our previous work [8, 9] and has the convenient features of short-range interactions, easily-variable wettability and a sharp liquid/vapor interface. Furthermore, its properties have been previously measured at this temperature: bulk fluid density $0.857 \sigma^{-3}$, viscosity $5.18 m /(\sigma \tau)$ and liquid-vapor surface 
tension $0.668 \epsilon / \sigma^{2}$.

For Couette flow a slab of liquid is placed between two solid plates, 15625 tetramer molecules in a cube of side $L=41.9 \sigma$ with 2916 solid atoms tethered in fcc layers above and below, and equilibrated for $100 \tau$. The LJ interaction parameters are set to be $c_{f f}=1$ and $c_{f w}=0.75$ for fluid-fluid and fluid-wall interactions, respectively. The bottom wall is then abruptly set into motion and translated steadily at velocity $U_{0}=0.1 \sigma / \tau$. The resulting velocity profile in the liquid at successive times is shown in the Fig. 1 at early $(10 \tau)$ and late $(200 \tau)$ times, and is seen to evolve into the expected linear form, with some velocity slip at the walls. The wall slip occurs because the fluid-wall interaction is weak: the interaction parameters used correspond to a partially wetting liquid with a sessile drop contact angle around $90^{\circ}$. The MD results are compared to a solution of the Stokes equation which incorporates the observed slip via a Navier boundary condition. This continuum flow field is obtained equivalently from a semi-analytic solutions in the literature [10] or from a direct numerical integration. The early-time agreement is only approximate, but this is not surprising given that we have imposed a shear stress instantaneously and examined the response after only about 20 ps. In this situation one expects some transient elastic or more generally non-Newtonian behavior in the fluid, and specifically a delayed response to the imposed shear. In the literature one finds, for example, Couette flow calculations using FENE-P dumbbell [11] which give a velocity profile which lags behind the Navier-Stokes solution at early times. Furthermore, in Fig. 1 $1 \mathrm{~b}$ we also show a good fit to the MD velocity profile using a simple shear-thinning model in which the viscosity varies as $\mu=\mu_{0} \cdot\left(1+\gamma / \gamma_{0}\right)^{-1 / 2}$, where $\gamma$ and $\gamma_{0}$ are the local and global strain rates. We have also reproduced the MD profile using a simple Maxwell model (not shown). The point of this discussion of the velocity profile is to argue that even for time-dependent flows, "experimental" MD results for velocity are consistent with the usual theoretical expectations for liquids, provided one takes account of inertia and a possible transient non-Newtonian response to abrupt changes in flow conditions.

We now make the analogous comparison for the forces the liquid exerts on the solid. In an MD calculation the force on any liquid atom is the simple sum of pairwise forces exerted by all other atoms within interaction range, so each wall force is the sum of forces between all fluid atoms and atoms in that wall, and can be isolated easily. The continuum force is just the shear stress times the wall area, $\pm L^{2} \mu \partial u / \partial y$, where the sign reflects the direction of the normal to the interface, $u$ and $x$ are the streamwise velocity and coordinate, and $y$ is the spanwise coordinate. The results for the lateral force on the two walls in Couette flow are displayed in Fig. 2, along with the corresponding continuum forces obtained from the Newtonian calculation incorporating slip. (The two nonNewtonian models mentioned above give similar results.) Several features are to be noted in this figure.

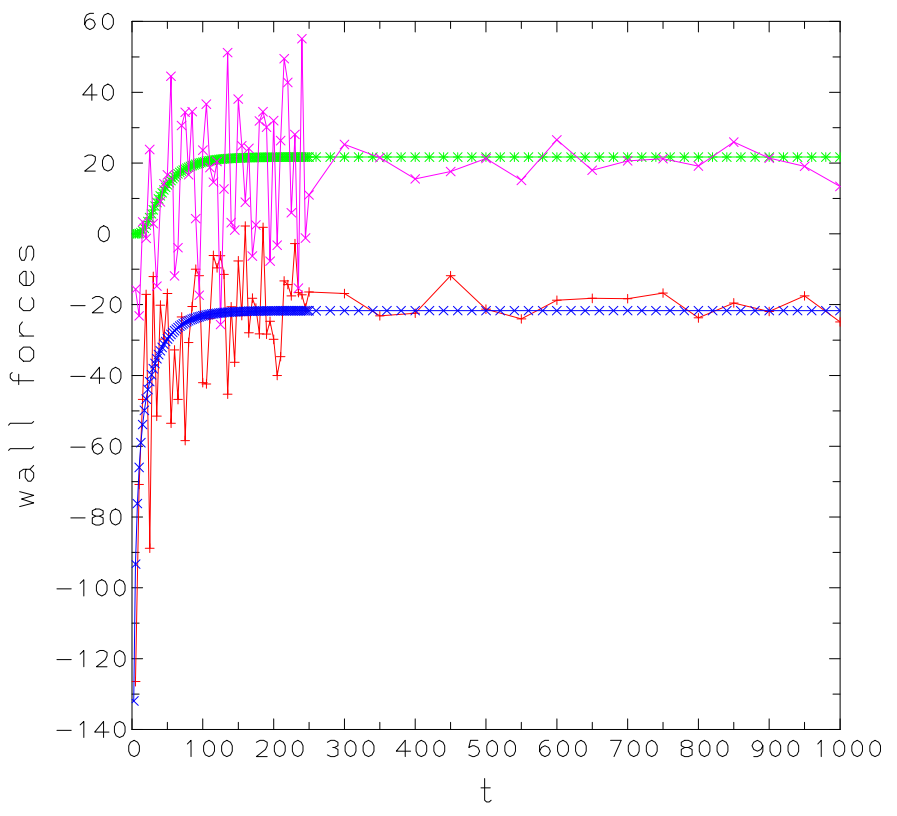

FIG. 2. Average wall forces in Couette flow. Bottom wall: MD results in red (+), Navier-Stokes in blue (x); top wall: MD results in green $(*)$. Navier-Stokes in magenta $(\mathrm{x})$.

(1) The MD force is noisy, because the intermolecular force is a rapidly varying function of interatomic spacing. The plotted curve is an average over 20 statistically independent realizations, obtained from different initial atomic velocity values, and even then a further time average is needed in order to identify the trend. The fluctuations are much more severe at early times $(t<200 \tau)$ because the data points are spaced by $5 \tau$ and the resulting averaging interval is short, while the data is more stable at later times where the interval is $50 \tau$.

(2) The continuum results are as expected. The lower wall is abruptly set into motion, giving a large stress at the start (nominally infinite) which relaxes to a constant value when the velocity profile stabilizes at linear. The liquid adjacent to the upper wall is at rest until the vorticity disturbance from the lower wall reaches it, so this force is initially zero and increases to its steady value as the fluid accelerates. In the steady state regime the wall forces are equal and opposite because the fluid is no longer accelerating and the forces must sum to zero. The force using the shear-thinning model is slightly different at early times but still in the middle of the MD fluctuations.

(3) The MD force agrees with the continuum force, modulo the fluctuations. The fluid begins to move immediately and the wall force does not exhibit stick-slip behavior or any analog of solid-on-solid static friction. The sharp peak in the force on the moving wall at early times results from inertia (included in the Newtonian continuum description) plus a bit of elasticity.

The main issue in this paper is the possibility of a 


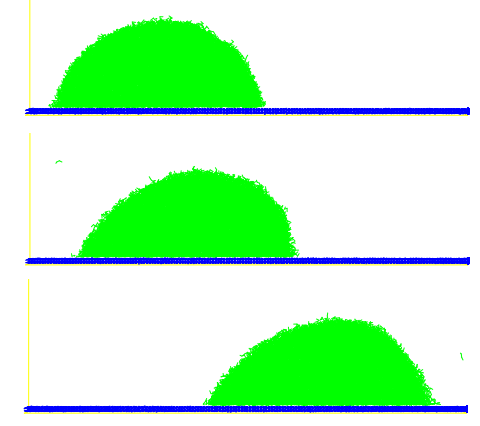

FIG. 3. Sessile drop driven across an atomically-smooth homogeneous substrate at times $0,500 \tau$ and $2500 \tau$ (top to bottom).

shear stress enhancement at the onset of motion, and in this configuration the cause is the abrupt motion of the wall. Indeed, the stress peak at $t=0$ is absent when the wall velocity is linearly ramped up from 0 to 0.1 , in place of the step change illustrated in the figure. Likewise, in a similar simulation of Poiseuille flow, even if a step pressure gradient is imposed the shear stress increases smoothly from zero. The distinction is completely accounted for by the Stokes equations: the analytic solutions for the velocity in start-up Couette and Poiseuille flow with a no-slip boundary condition, given for example in [12, directly indicate whether a stress peak is present. These solutions involve a Fourier series which converges for the shear stress at the bottom wall at $t=0$ for Poiseuille flow but diverges in the Couette case.

\section{SESSILE DROPS}

The simplest fluid mechanical analog of solid-solid friction involves a drop sliding on a solid surface. Although these is an extensive literature on this problem [13, 14] relatively little attention has been given to the forces involved, and we use MD simulations to disentangle them. We begin with a partially-wetting liquid on a uniform and atomically-smooth substrate, in the form of cylindrical cap drop shown in Fig. 3 . The advantage of this shape compared to a spherical cap is that the system is statistically homogeneous in the direction $(z)$ normal to the plane of the figure, and the results can be averaged over $z$. The wall-fluid interaction strength is chosen as $c_{f f}=0.85$ which gives an initial contact angle of $70^{\circ}$. After equilibration a constant force $f=0.001 \mathrm{~m} \sigma / \tau^{2}$ is applied to each atom and the drop translates, as shown in the subsequent frames of the figure. The Reynolds number, $R e \equiv \rho \dot{X} H_{0} / \mu$ where $H_{0}$ is the equilibrium drop height and $\dot{X}$ is the center of mass velocity (see below), is 0.0748 and the Capillary number $C a \equiv \mu \dot{X} / \gamma$ is 0.149 , with slightly different values in the other cases. As the drop moves its shape changes and exhibits distinct advancing and receding dynamic contact angles which, as
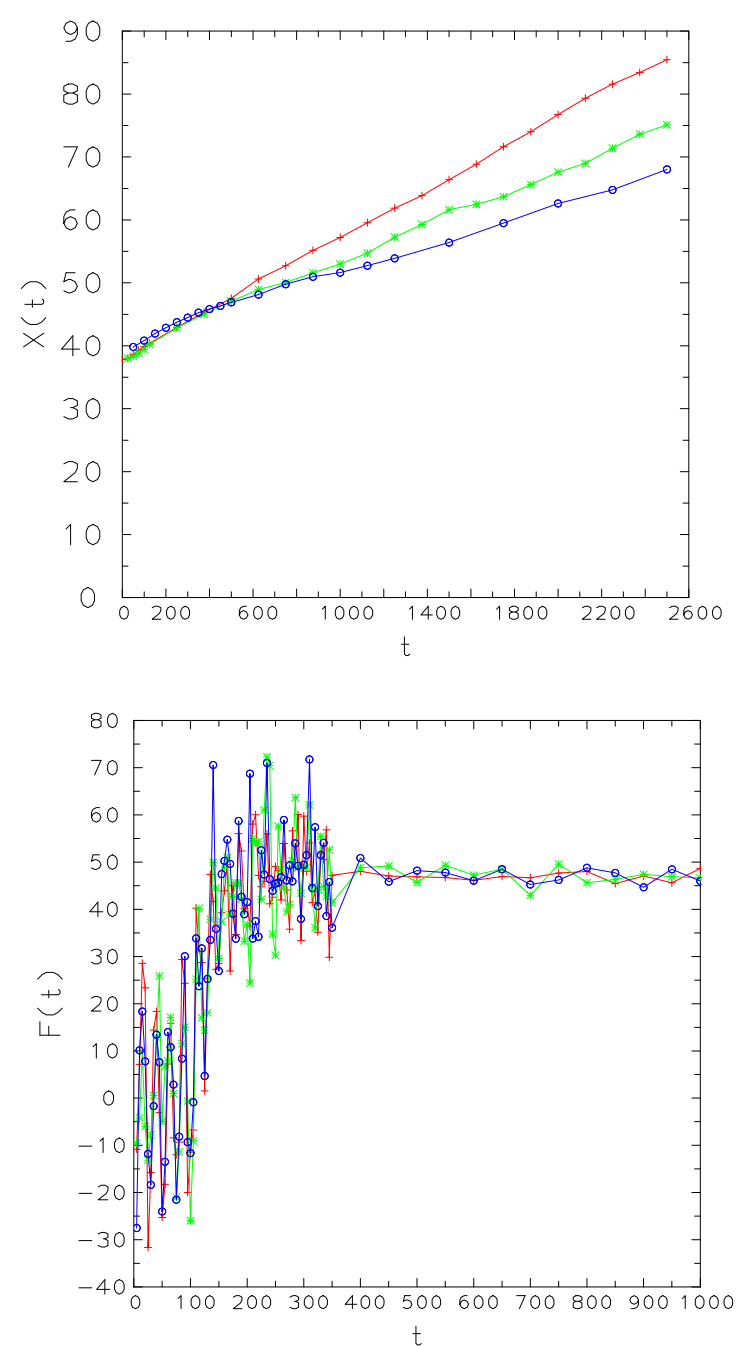

FIG. 4. Center of mass (top) and lateral force (bottom) vs. time for a sessile drop forced across the various substrates at $f=0.001$, averaged over 20 realizations. Smooth: red $(+)$, chemical step: green $(*)$, physical step: blue $(\circ)$.

seen in Fig. 3. fluctuate during the motion. A constant external force is formally equivalent to tilting the substrate in the presence of gravity, although realistically the values of $f$ used here are much too large for this interpretation. The behavior is similar over a range of forcing values near this one, $5 \times 10^{-5}$ to 0.005 , but for significantly lower values the force on the drop is unmeasurable because the signal is swamped by the fluctuations and the drop motion itself becomes intermittent. As for higher forcing values, at $f=0.01$ the center of mass still moves with constant velocity although the drop becomes increasingly elongated and at still higher values the drop tends to fly off the substrate.

Most realistic solid surfaces are heterogeneous and irregular, and we can address these complications by forcing the drop across a modified substrate involving either a chemical or a physical heterogeneity. The drop is equi- 


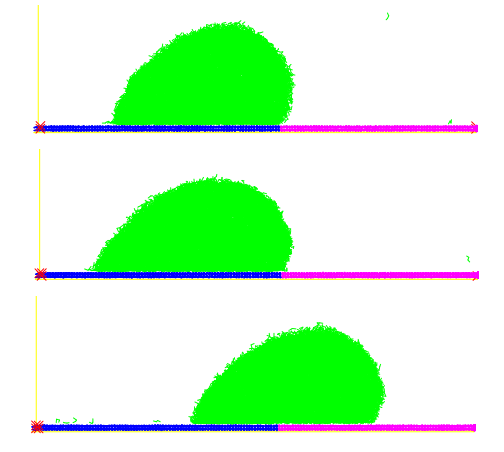

FIG. 5. Sessile drop driven across an substrate with a chemical step. Pinned at $f=0.0005$ (top) and in motion for $f=0.001$ at times $500 \tau$ (center) and $2500 \tau$ (bottom).

librated as before but to the right of the drop there is either a chemical step where the wettability coefficient $c_{f w}$ drops to 0.65 or a physical step where the height of the solid rises by one fcc unit cell, a distance of $1.17 \sigma$ here. When a lateral force is applied the drop tilts in the direction of the force, developing distinct advancing and receding angles, $\theta_{A, R}$, but moves only if the force is large enough. We illustrate this behavior in Fig. 5f for the chemical step case, where the value of the threshold force needed to move the drop over the step can be obtained by a the following force balance [2].

In equilibrium on a homogeneous surface a drop is held in place by solid/liquid, solid/vapor and liquid/vapor surface tension forces at the contact line, with a lateral force balance given by Young's equation $\gamma_{S V}-\gamma_{S L}=$ $\gamma \cos \theta_{0}$. For non-ideal surfaces there is a range of possible equilibrium contact angles. If a weak force $f$ is applied to each atom, a drop tilts in the direction of the force but can remain at rest if the applied force is balanced by the unbalanced surface tension forces per length $\gamma\left(\cos \theta_{R}-\cos \theta_{0}\right)$ at the receding contact line and similarly at the advancing line. Explicitly, the force balance is $f N=\gamma W\left(\cos \theta_{R}-\cos \theta_{A}\right)$, where $N=46800$ is the number of atoms in the drop and $W$ length of the contact line (the width of the drop in this situation, $51.3 \sigma$ ). For the chemical step, we observe that the drop appears stationary at force 0.0005 , where it is pinned at the edge of the step, but moves steadily at 0.0006 and higher values. Using the drop density profile at 0.0005 and defining the liquid/vapor interface to be the contour where the density is half the bulk value, we estimate $\theta_{R} \approx 67^{\circ}$ and $\theta_{A} \approx 111^{\circ}$, giving $f=0.00055$ from the force balance equation, in agreement with the simulation. A similar transition and corresponding force balance is found in the case of a physical step. If there are no angles $\theta_{A, R}$ in the equilibrium range satisfying the force balance the drop will move, and in the present simulations moving drops are expected to have have constant velocity, characteristic of linear friction in Stokes flow.

We observe that, despite the shape fluctuations seen in the figures, the motion is steady: for forcing $f=0.001$
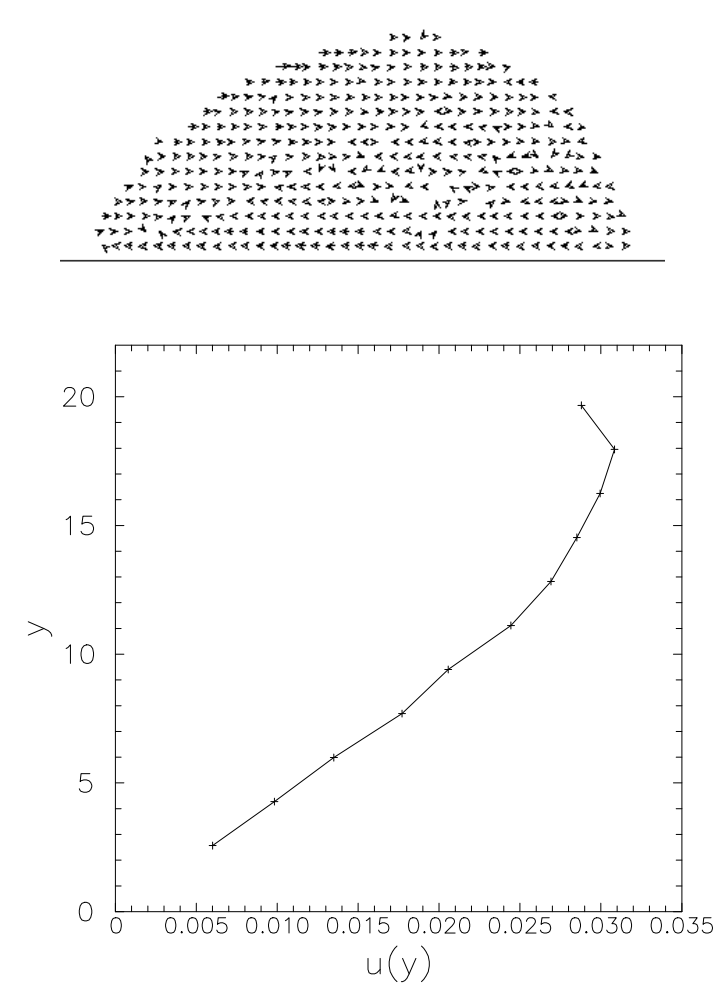

FIG. 6. Flow inside the drop on a homogeneous substrate. Two-dimensional velocity field in the moving center of mass frame (top) and average lateral velocity profile in the center of the drop in the substrate frame (bottom).

Fig. 4 shows the drop center of mass $X(t)$ moving with nearly constant velocity from the start for both smooth and heterogeneous surfaces. The total lateral force $F(t)$ the liquid exerts on the wall at $f=0.001$ is given as a function of time in Fig. 4 in all cases the wall force oscillates about zero before the external force is applied at time $100 \tau$ and afterwards ramps monotonically up to to a plateau. Only the transient behavior immediately after application of the external force shows a variation with the structure of the substrate. The distinction between the size of the fluctuations at early and late times is the same as in the previous Couette simulations: short and long averaging intervals, respectively. The average force in the plateau fluctuates about the value of the net force applied to the liquid, $N f=46.8$, as expected because the drop is not accelerating. It is possible to decompose the wall force into capillary forces at the contact line plus frictional drag on the drop, but unfortunately the dynamic contact angles could not be measured with any accuracy, and we have not pursued this.

The motion of the liquid inside the drop is a combination of rolling and slipping, as indicated in Fig. 6 for the smooth wall. The two-dimensional flow field shown is an average over $50 \tau$ and evaluated in a reference frame moving with the drop center of mass; the resolution is poor since the velocities involved are $O\left(10^{-2}\right)$ times the 
random thermal velocity and the center of mass velocity is not exactly constant during the averaging interval, but rotation about the middle of the drop is evident. Ensemble averaging tends to wash out the result for this two-dimensional field, but is more effective for the velocity profile, which is instead evaluated in the reference frame of the solid wall and is also an average over the middle of the drop. The result is a roughly linear profile as would correspond to rotation about the wall. (The uppermost points correspond to the liquid/vapor interfacial region where the density is falling off.) The presence of slip at the wall requires a small discussion. The region $0 \leq y \leq 1.71 \sigma$ is occupied by solid (at rest), and the lowest liquid point (at $y=2.565$ ) is in the center of a finite-sized sampling bin in the liquid. The precise definition of the "solid wall" is always ambiguous at atomic resolution, but would certainly be somewhere in the region $1.71 \sigma \leq y \leq 2.5 \sigma$, where $u$ is non-zero. Aside from this ambiguity, as in Couette flow the presence of slip varies with the strength of the fluid-wall interaction but the rolling motion is always present.

The force exerted on the drop by the walls, can be examined locally by computing the force as a function of the coordinate along the base of the drop. We use a sequence of slabs of wall, $i * d x<x<(i+1) * d x$ and $0<y<L$ with $d x=1.71 \sigma$, and compute the force per area on each slab. (Note that this quantity is almost the $x$ - $y$ component of the fluid's shear stress tensor at the wall. The qualification is because, as in the slip discussion above, MD fields are always averages over a finite-sized sampling bin with the result assigned to its center, which is displaced from the wall by half the bin size. A further extrapolation would be required to determine the force at the wall.) In Fig. 7 we show the horizontal $(x)$ and vertical $(y)$ components of the force on the drop, along with the local density and lateral velocity, both in equilibrium and while translating. The forces peak at the drop's two contact lines and are constant on average in the interior. In the $x$-direction, the signs of the two peaks correspond to the fact that the liquid/vapor surface tension acts to contract the drop into to a circular cylinder but the attraction to the wall draws the edges of the drop outwards, corresponding to an inward force on the wall. The wall force in the interior of the drop is (statistically) constant, zero when the drop is in equilibrium and positive during drop translation due to the liquid pulling to the right. In the $y$-direction, the force peaks at the contact lines, again correspond to surface tension trying to contract the drop by pulling upwards on the wall, while in the interior the force is a constant, corresponding to the Laplace pressure inside the drop.

A similar but more detailed discussion of the variation of these local forces with contact angle and a comparison to Young's equation is given by Fernandez-Toledano, et al., [15] for a different configuration involving a liquid bridge in equilibrium between two solid plane walls. In that case the drop is confined by the walls but here is it is only attracted to one wall, so the force analysis differs
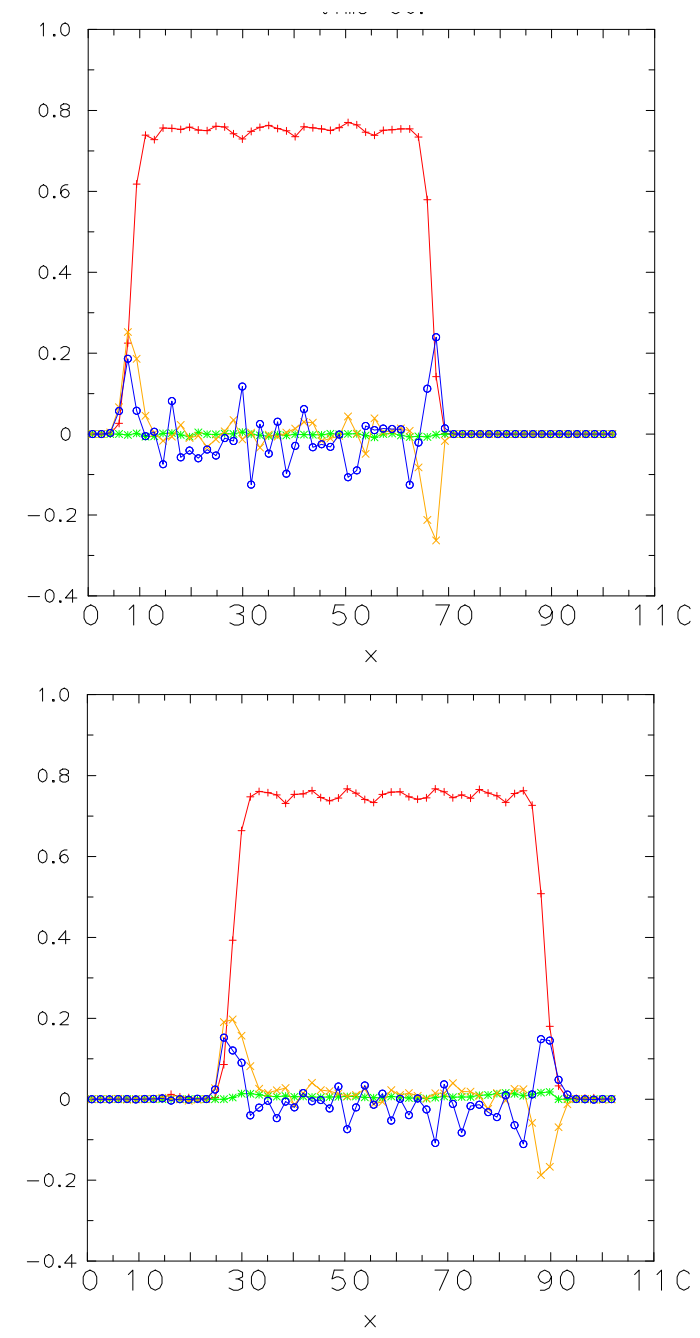

FIG. 7. Local fields for a drop on a homogeneous substrate, at rest (top) and in motion (bottom) after $250 \tau$, in a single realization. Density: red $(+), x$-velocity: green $(*), x$-force: orange (x) and $y$-force: blue (o).

in detail. The principal point we wish to make here is that when the drop moves the numerical values change but there is no qualitative difference in local forces on the wall. The density is constant inside the drop, and its profile simply shifts in $x$ as the drop moves, while the liquid velocity at the wall is constant, zero in equilibrium and approximately equal to the center of mass velocity during the motion.

\section{CORNERED DROPS}

The experiments which motivated this study involved spherical drops on a sliding stage held in place by a measurement pin, and includes features of both of the previous simulations. The liquid is forced into motion abruptly as in Couette flow, with a localized impulsive stress at a solid boundary, and then "slides" relative to 


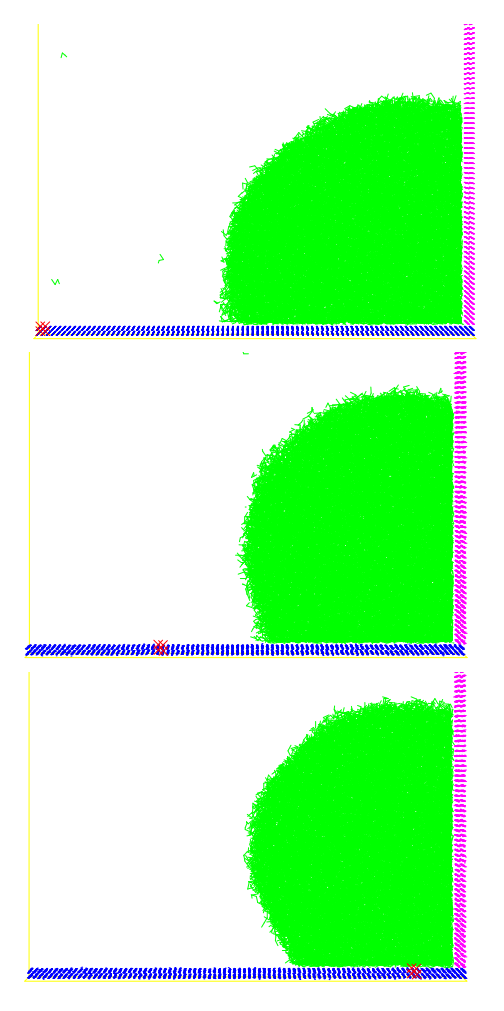

FIG. 8. Drop driven into a corner by motion of the bottom wall to the right, at times $0,200 \tau$ and $600 \tau$ (top to bottom). The translation of the wall is shown by the red dot which indicates the location of an atom fixed in the wall,

the solid with (as we shall see) a combination of slip and rolling. Again we take advantage of MD's direct access to the force and use a simpler configuration with no variation in one direction to improve the statistics.

We place a drop in the corner formed by two distinct solid walls meeting at a right angle, as shown in Fig. 8 , and choose the (partial) wetting coefficient $c_{f f}=0.75$. The simulation box is a cube of side $68.4 \sigma$ containing 16,000 tetramer molecules, which initially fill an approximate circular quadrant with a contact angle of $93^{\circ}$. The vertical wall is fixed in place while the horizontal wall is, after equilibration, translated towards the fixed wall at a constant velocity. Due to periodicity, the sliding wall can translate indefinitely. The gap where the two walls meet is the same size as the internal wall lattice spacing, and there is no interaction between the respective wall atoms, and therefore no fluid leakage or distortion of the corner or any solid frictional heat generated there. These simplifying properties would be difficult to realize in a laboratory experiment, although the differences involve only the corner region which is not the focus of this study in any case.

We consider a range of wall velocities 0.01 to $1.0 \sigma / \tau$. At the lower velocities we use the same time step and wall tethering strength as in the previous simulations, but for the higher velocities the time step is lowered to $0.001 \tau$ and instead of tethering thermally active wall atoms, the
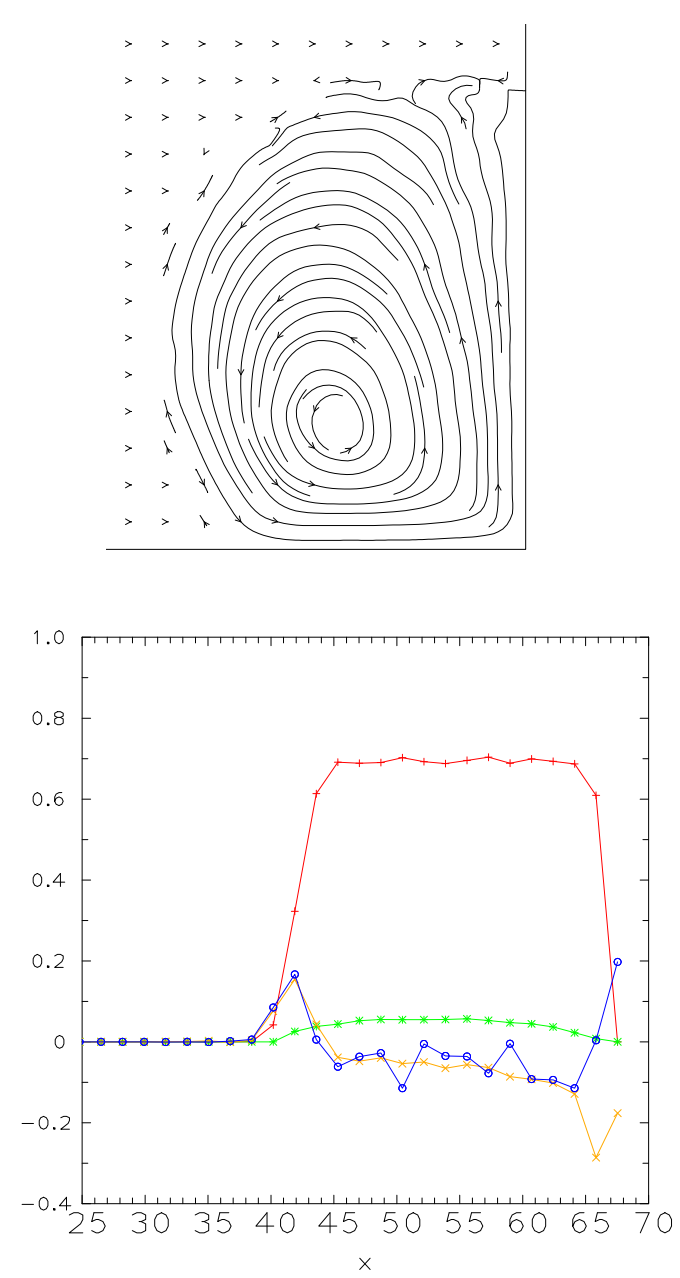

FIG. 9. Streamlines (top) and local fields (bottom) for a drop squeezed into a corner at $u=0.1$ : density: red $(+)$, $x$-velocity: green $\left(^{*}\right), x$-force: orange $(\mathrm{x})$ and $y$-force: blue (o).

wall is translated as a rigid (but slightly randomized) lattice. The reason is that at higher velocities the atom positions lag significantly behind the tether positions unless a very high binding force is used, which would require a correspondingly very small time step to resolve. We have verified that the fluid motion and wall forces are not sensitive to this modification. When the lower wall moves (right), the drop is squeezed into the corner, as shown in Fig. 8 for the $u_{w}=0.1$ case, and evolves to a roughly time-independent configuration after several hundred $\tau$. In the steady state, the advancing contact line at the horizontal wall (in the rest frame of this wall the contact line advances to the left) increases to a velocity-dependent value $\left(100\right.$ to $\left.145^{\circ}\right)$, while the static angle on the vertical wall shows little change. The motion inside the drop is rolling, as seen in Fig. 9, accompanied by some slip at the wall. The horizontal force the liquid exerts on the on the wall is negative, opposing the wall motion, with a peak at the corner where a continuum no-slip flow field would have a stress singularity, while the vertical force 

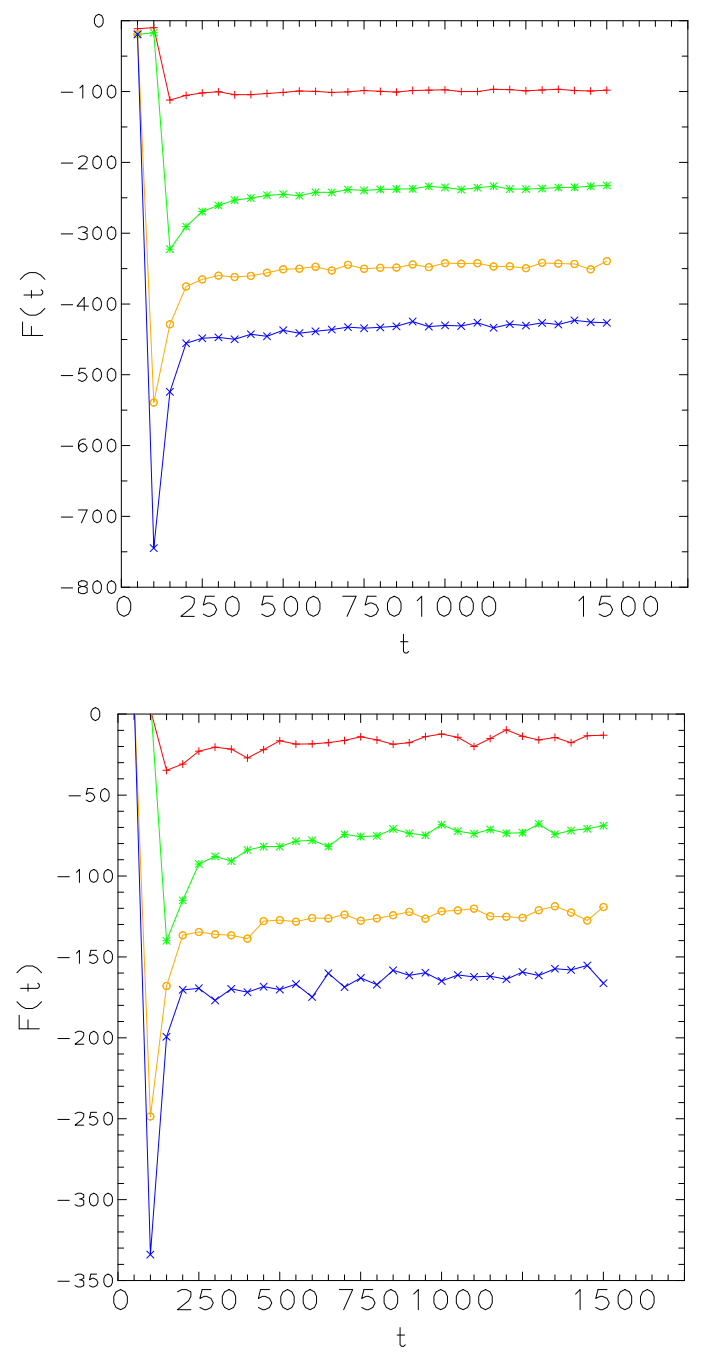

FIG. 10. Time variation of the $x$ (top) and $y$ (bottom) force on the sliding wall for various velocities. $u=0.1$ : red $(+)$; $u=0.25$ : green $\left(^{*}\right) ; u=0.5$ : orange (o) and $u=1.0$ : blue $(\mathrm{x})$.

has the same interpretation as for sessile drops. At higher wall velocities, the liquid density profile becomes slightly asymmetric and larger near the corner, the slip velocity increases, also asymmetrically but instead largest at the contact line, and the force profiles increase in magnitude but maintain the same shape.

The $x$ and $y$ components of the total force on the bottom wall are shown in Fig. 9, for different values of the wall velocity. In all cases there is an initial peak, resembling that appearing in Couette flow, which we attribute to the inertial effects of an impulsive start. The reason is that the duration of the stress peaks is $100-150 \tau$ (note that wall motion begins at $100 \tau$ ) which is comparable to the vorticity diffusion time across the drop, $R^{2} / \mu$ with drop size $R \sim 35 \sigma$ and viscosity $\mu=0.518 m /(\sigma \tau$, which characterizes flow development. The signs of the fluid forces on the wall are negative because the walls are

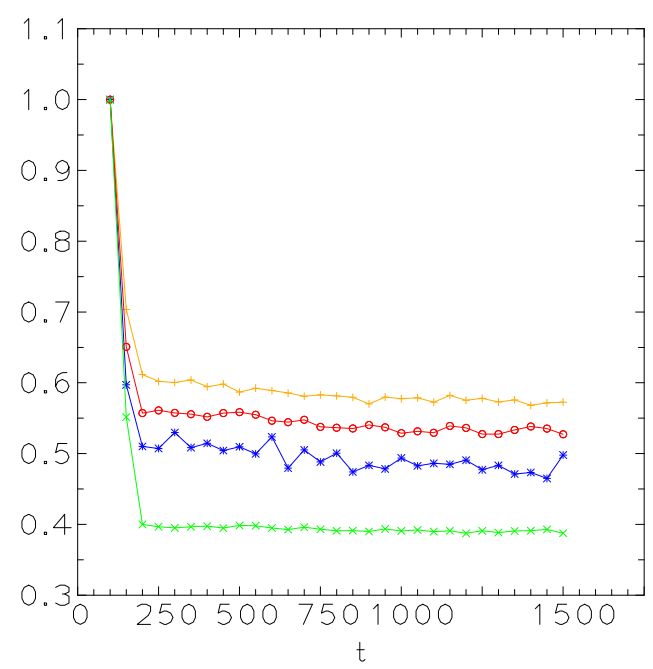

FIG. 11. Time decay of fluid density and velocity near the wall, and the $x, y$ forces on the drop at $u=1.0$; color code as in Fig. 7

squeezing the fluid to the right and upwards and the fluid resists this.

The other notable feature of the forces is the systematic weak decay (towards zero) following the peak, surprising since one would have expected constant wall forces in an apparently steady flow. However, in this situation the drop is not confined and is free to adjust its position. intuitively one might imagine the drop pushed upwards by repeated collisions with the sliding wall atoms and in fact precisely this behavior is observed. In Fig. 11 we plot the density and velocity in the fluid adjacent to the wall at low and high velocities, normalized to their values at time when the wall begins to move. The initial peak results from the abrupt start, following which there is a weak decay of the density, meaning fluid moves away from the wall, which produces parallel decays in the fluid velocity and wall forces.

\section{THE EXPERIMENT}

There remains the question of interpreting the experiments of Gao et al. The principal distinction between that experiment and the drop simulations here is the distortion of the drop surface as it moves relative to the measuring pin. Rearrangement of the liquid drop's shape and the contact lines on the pin and on the substrate would certainly affect the force exerted We have carried out several simulations on this process, involving a spherical cap drop and a finite-sized obstacle pin, rather than a barrier wall as in the previous section, but these simulations do not completely reproduce the experiments in terms of drop distortion and wall force.

An example is given in Fig. 12 - a spherical cap is placed on a partially wetting substrate $\left(c_{f f}=0.85\right)$ and 


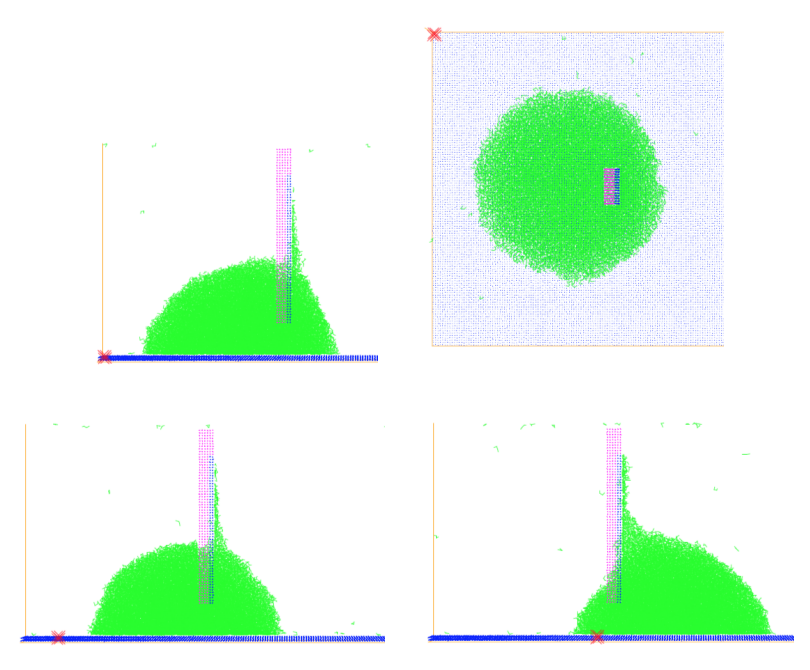

FIG. 12. Simulation of the experiment of Gao et al. 44. Top: side and top view of the equilibrated system, Bottom: effects of sliding the substrate to the left, at times 1000 and $5000 \tau$.

equilibrated with a rectangular pin inserted in the drop from above. Most of the pin has the same interaction as the substrate, except that the upstream face is more strongly wetting $\left(c_{f f}=1.2\right)$, in order to mimic the experiment where a metallic reflecting layer was added to improve the imaging. After equilibration the substrate is translated to the right at velocity $u=0.1$ and the force on the pin recorded. Fig. 13 again shows a monotonic rise to a near-plateau, with no "static-friction" enhancement. At times beyond $5000 \tau$ the drop becomes highly elongated and eventually detaches from the pin, but during the interval indicated the footprint of the drop on the substrate remains approximately circular. The results are similar when the conditions of the simulation are varied (different wettabilities, different pin shapes, different speeds, etc.), and provided the pin remains embedded inside the drop the force on the pin is roughly constant. Exceptions to the typical behavior are found when the pin is at the edge of the drop, when the liquid either first wets or dewets the pin. In these cases the liquid/vapor interface does deform and a transient peak or spike appears in the force.

In contrast to the simulations, the experimental drops do change shape and, in particular, their contact line length increases during the time interval when the force is enhanced. A further distinction in the simulations, a consequence of size limitations, is that the liquid climbing the wetting side of the pin has a density corresponding to liquid/vapor interfacial region and does not faithfully represent bulk liquid and may not exert the proper hydrodynamic drag. Nonetheless, it appears that the transient friction enhancement in the experiments is related to change of drop shape, and is not a general character- istic of solid/liquid friction.

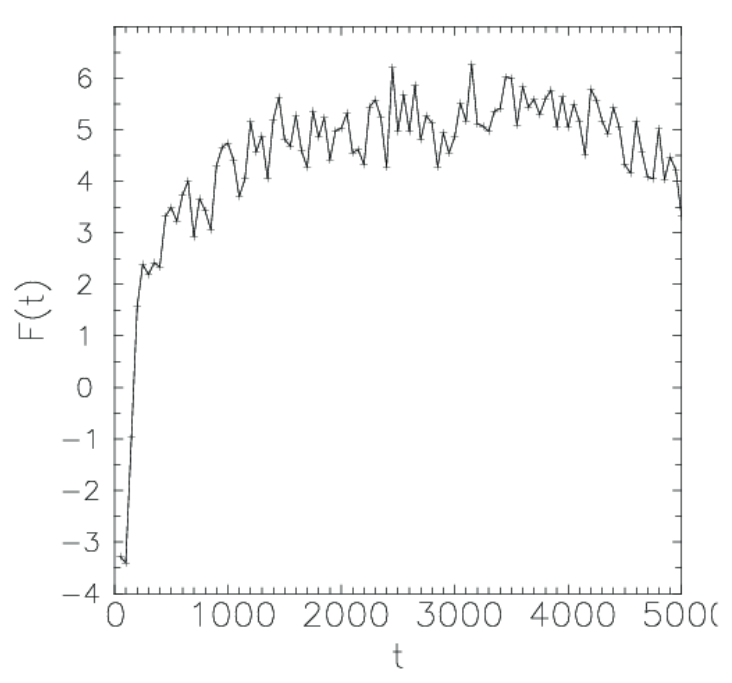

FIG. 13. Force on the pin in the simulation shown in Fig. 12

\section{CONCLUSIONS}

We have used MD simulations to investigate the possibility of an enhanced shear stress before a liquid begins to move across a solid surface, analogous to the distinction between static and dynamic friction when two solids move relative to each other. In cases where the motion is driven by a constant body force applied to the interior of the liquid, even in a step-wise fashion, the stress is found to increase monotonically from zero to a steady state value. An abrupt motion of a solid bounding surface, however, generates a large local strain which in turn produces a peak in the shear stress. Such peaks are infinite in the (mathematical) continuum limit but regularized to large but finite values in MD simulations and in real life, but otherwise entirely in accord with the NavierStokes equations. Furthermore, even in situations where the liquid is pinned by inhomogeneities and requires a minimum threshold force for continuous motion, no force enhancement is found. The experiments [4] which motivated this work appear to incorporate changes in drop shape and thereby involve interfacial dynamics as well as wetting considerations, and do not provide evidence for an analog of static solid/solid friction. 
[1] J.C. Anderson, K.D. Leaver, R.D. Rawlings and J.M. Alexander, Materials Science, 4th ed., (Springer, New York, 1990).

[2] P.-G. de Gennes, F. Brochard-Wyart and D. Quéré, Capillarity and Wetting Phenomena: Drops, Bubbles, Pearls, Waves (Springer, New York, 2004).

[3] B. N. J. Persson, Sliding Friction, 2nd ed. (Springer, New York, 2000).

[4] N. Gao, F. Geyer, D. W. Pilat, S. Wooh, D. Vollmer, H.J. But and R. Berger, How drops start sliding over solid surfaces, Nature Phys. 14, 191 (2018).

[5] M. P. Allen and D. J. Tildesley, Computer Simulation of Liquids (Clarendon, Oxford, 1987).

[6] D. Frenkel and B. Smit, Understanding Molecular Simulation, 2nd ed. (Academic, San Diego, 2002).

[7] G. S. Grest and K. Kremer, Molecular dynamics simulation for polymers in the presence of a heat bath, Phys. Rev. A 33, 3628 (1986).

[8] T. S. Lo, J. Koplik, M. Rauscher and S. Dietrich, Pearling instability of nanoscale fluid flow confined to a chemical channel, Phys. Fluids 18, 031104 (2006).

[9] J. Koplik and C. Maldarelli, Diffusivity and hydrodynamic drag of nanoparticles at a vapor-liquid interface, Phys. Rev. Fluids 2, 024303 (2017).

[10] G. Kaoullas and G. C. Georgiou, Start-up and cessation Newtonian Piseuille and Couette flows with dynamic wall slip, Meccanica 50, 1747 (2015).

[11] Y. Mochimaru, Unsteady-state development of plane Couette flow for viscoelsatic fluids, J. Non-Newtonian Fluid Mech. 12, 135 (1983).

[12] C. Pozrikidis, Introduction to Theoretical and Computational Fluid Dynamics (Oxford, New York, 1997).

[13] D. Bonn, J. Eggers, J. Indekeu, J. Meunier and E. Rolley, Wetting and spreading, Rev. Mod. Phys. 81. 739 (2009).

[14] Special section containing papers on the dynamics of wetting, J. Phys.: Condens. Matter, 21, 464117 (2009).

[15] J.-C. Fernandez-Toledano, T. D. Blake, P. Lambert and J. De Coninck, On the cohesion of fluids and their adhesion to soilds, Adv. Colloid Interface Sci. 245, 102 (2017). 